\title{
The role of B cells in metabolic (dysfunction)-associated fatty liver disease
}

\author{
Ziyan Pan ${ }^{1}$, Wah-Kheong Chan ${ }^{2}$, Mohammed Eslam ${ }^{1}$ \\ ${ }^{1}$ Storr Liver Centre, Westmead Institute for Medical Research, Westmead Hospital and University of Sydney, Sydney, Australia; ${ }^{2}$ Gastroenterology \\ and Hepatology Unit, Department of Medicine, Faculty of Medicine, University of Malaya, Kuala Lumpur, Malaysia \\ Correspondence to: Mohammed Eslam. Storr Liver Centre, Westmead Institute for Medical Research, Westmead Hospital and University of Sydney, \\ Sydney 2145, Australia. Email: mohammed.eslam@sydney.edu.au. \\ Comment on: Barrow F, Khan S, Fredrickson G, et al. Microbiota-Driven Activation of Intrahepatic B Cells Aggravates NASH Through Innate and \\ Adaptive Signaling. Hepatology 2021;74:704-22.
}

Submitted Sep 27, 2021. Accepted for publication Oct 18, 2021.

doi: 10.21037/hbsn-21-404

View this article at: https://dx.doi.org/10.21037/hbsn-21-404

Metabolic (dysfunction)-associated fatty liver disease (MAFLD; formerly known as non-alcoholic fatty liver disease) is the most common liver disorder, affecting around one-third of the population worldwide (1). MAFLD is a heterogeneous disease with a spectrum of liver pathologies that spans from hepatic lipid accumulation (steatosis) to chronic inflammation (steatohepatitis), which can progress to cirrhosis and hepatocellular carcinoma. It is impacted by a myriad of factors, including metabolic health, biological and chronological age, genetics and epigenetics (2-4). However, only a proportion (5-40\%) of patients develop liver inflammation or steatohepatitis (5). This transition is a cardinal feature of progressive liver disease, which is the precursor to the development of the hepatic and extrahepatic outcomes.

Notably, the unique anatomical structure and position of the liver, where immune cells encounter antigen-rich blood from the gastrointestinal tract, have rendered it to be considered as an immunological organ. The recruitment and migration of immune and pro-inflammatory cells into the liver, which subsequently profoundly shapes the immunological response of the liver, is a crucial event in the initiation and progression of the critical transition to steatohepatitis (6). In this complex microenvironment, innate immune mechanisms, which include the activation of resident Kupffer cells and recruitment of leukocytes that release inflammatory mediators and contribute to the inflammatory response, have long been thought as the main contributing factor to the progression to steatohepatitis (6). However, emerging evidence suggests that adaptive immunity could play a pivotal role in this process. As B lymphocytes produce cytokines and antibodies, with recent evidence suggests their crucial role in promoting inflammation and the progression of MAFLD, although this remains to be fully characterised.

In a recent elegant study, Barrow et al. (7) provided novel insights into this role of B lymphocytes. In their work, they suggested a pivotal role of B cells as well as the communication between $B$ cells and other intra-hepatic both immune and non-immune cells in the progression of MAFLD. They found that B cells are among the most abundant immune cells in the liver and are strong inducers of T-cell responses during the progression of steatohepatitis. They demonstrated that B-cell depletion led to a net effect of resolution of steatohepatitis, while adoptively transferring B cells from NASH livers caused disease recurrence, suggesting that B-cell activation in the liver is a causative factor rather than a bystander in the pathogenesis of steatohepatitis.

Broadly, according to the expressions of cell-surface markers, B cells can be classified into two subsets (B1 and B2). B1 cells arise from the foetal liver, while B2 cells derive from haematopoietic progenitors in the bone marrow (8). Barrow et al. (7) reported that high-fat high-carbohydrate diet-fed mice that mimicked various features of human steatohepatitis demonstrated an enhanced prevalence of B2 cells and a decreased percentage of B1b cells compared with normal chow diet-fed mice. In mice with steatohepatitis, this $\mathrm{B}$ cell response is accompanied by the upregulation of hepatic expression of B cell-activating factor (BAFF), one of 
the cytokines that promotes B cell survival and maturation (9). Interestingly, circulating levels of BAFF has been shown to be higher in patients with steatohepatitis than in those with simple steatosis, and the levels correlated with the severity of steatohepatitis and fibrosis (10).

The pro-inflammatory and fibrogenic roles of B cells in MAFLD involve their interaction with other immune and non-immune hepatic cells. Barrow et al. (7) reported that B cells secrete fewer inflammatory cytokines than macrophages and neutrophils on a per-cell basis. They found that B cells in steatohepatitis livers stimulate the generation of CD4 and CD8 T cells, which promote inflammatory response. In addition, these inflammatory mediators might be implicated in the activation of hepatic stellate cells and liver macrophages. Of note, Barrow et al. (7) observed that intra-hepatic, but not splenic, B cells stimulate type 1 T-helper (Th1) responses. As indicated by the authors, these findings suggest that the B-cell acquisition of the proinflammatory phenotype is likely secondary to local activation rather than a systemic inflammatory response.

In both human and murine models, the 'Western' diet has been found to induce alterations in the gut flora and intestinal permeability, which lead to increased circulating levels of antigen-rich bacterial products. Subsequent intra-hepatic activation of pattern recognition receptors, including toll-like receptors, a sensor for these products, is a pivotal event in the initiation of steatohepatitis (6). Differential gut microbiota between subtypes of MAFLD has been suggested (11). Barrow et al. (7) also reported that the mechanisms of $\mathrm{B}$-cell activation include gut-derived microbial antigens that may serve as ligands that activate intra-hepatic B cells during steatohepatitis. They also suggested that B-cell activation during steatohepatitis can occur simultaneously through the innate adapter myeloid differentiation factor 88 (MyD88) signalling, which leads to the stimulation of the nuclear factor $\mathrm{\kappa B}$ and $\mathrm{B}$-cell receptor (BCR) signalling pathways. This was based on the finding that $\mathrm{B}$ cell-specific deletion of MyD88 ameliorated hepatic T cell-mediated inflammation and fibrosis (7).

As is typical in science, this work raises some other questions and stimulates further directions. Future research is needed to identify additional critical contextual mechanisms, both antigen dependent and independent, for the activation of intra-hepatic B cells and enhancing the progression to steatohepatitis. In addition, identification of B-cell-directed therapies can be an attractive therapeutic avenue. Various strategies may be employed to achieve this goal including B-cell depletion, suppression of B-cell survival and proliferation, attenuation of the interaction between $\mathrm{T}$ and $\mathrm{B}$ cells and modulation of $\mathrm{B}$-cell downstream inflammatory mediators or BCR signalling pathways (12). However, untargeted side effects, including toxicity and immunosuppression, may be a concern. Therefore, further preclinical evidence, including the delineation of the differential role of various B-cell subsets in the pathogenesis of MAFLD, is needed to guide the development of moreselective strategies in targeting the B-cell signalling pathway for the treatment of MAFLD.

In summary, the study by Barrow et al. (7) highlights that B cells are an emerging central player in MAFLD progression. This finding provides interesting novel clues into the role of immune cells in the pathogenesis of the disease and therefore represents a new avenue for the potential effectiveness of B-cell-specific therapeutic strategies.

\section{Acknowledgments}

Funding: Mohammed Eslam is supported by the Robert W. Storr Bequest to the Sydney Medical Foundation, University of Sydney; National Health and Medical Research Council of Australia (NHMRC) Program Grants (1053206 and 1149976) and Project grants (1107178 and 1108422, 2001692).

\section{Footnote}

Provenance and Peer Review: This article was commissioned by the editorial office, Hepatobiliary Surgery and Nutrition. The article did not undergo external peer review.

Conflicts of Interest: All authors have completed the ICMJE uniform disclosure form (available at https://hbsn. amegroups.com/article/view/10.21037/hbsn-21-404/coif). Dr. ME serves as an unpaid editorial board member of Hepatobiliary Surgery and Nutrition. Dr. WKC has served as a consultant for Boehringer Ingelheim and Novo Nordisk; and a speaker for Novo Nordisk, Viatris and Hisky Medical. The other author has no conflicts of interest to declare.

Ethical Statement: The authors are accountable for all aspects of the work in ensuring that questions related to the accuracy or integrity of any part of the work are appropriately investigated and resolved.

Open Access Statement: This is an Open Access article distributed in accordance with the Creative Commons 
Attribution-NonCommercial-NoDerivs 4.0 International License (CC BY-NC-ND 4.0), which permits the noncommercial replication and distribution of the article with the strict proviso that no changes or edits are made and the original work is properly cited (including links to both the formal publication through the relevant DOI and the license). See: https://creativecommons.org/licenses/by-nc-nd/4.0/.

\section{References}

1. Sarin SK, Kumar M, Eslam M, et al. Liver diseases in the Asia-Pacific region: a Lancet Gastroenterology \& Hepatology Commission. Lancet Gastroenterol Hepatol 2020;5:167-228.

2. Petta S, Eslam M, Valenti L, et al. Metabolic syndrome and severity of fibrosis in nonalcoholic fatty liver disease: An age-dependent risk profiling study. Liver Int 2017;37:1389-96.

3. Bayoumi A, Grønbæk H, George J, et al. The Epigenetic Drug Discovery Landscape for Metabolic-associated Fatty Liver Disease. Trends Genet 2020;36:429-41.

4. Eslam M, George J. Genetic Insights for Drug Development in NAFLD. Trends Pharmacol Sci 2019;40:506-16.

5. Eslam M, Sarin SK, Wong VW, et al. The Asian Pacific

Cite this article as: Pan Z, Chan WK, Eslam M. The role of $\mathrm{B}$ cells in metabolic (dysfunction)-associated fatty liver disease. HepatoBiliary Surg Nutr 2021;10(6):875-877. doi: 10.21037/ hbsn-21-404
Association for the Study of the Liver clinical practice guidelines for the diagnosis and management of metabolic associated fatty liver disease. Hepatol Int 2020;14:889-919.

6. Alharthi J, Latchoumanin O, George J, et al. Macrophages in metabolic associated fatty liver disease. World $\mathrm{J}$ Gastroenterol 2020;26:1861-78.

7. Barrow F, Khan S, Fredrickson G, et al. MicrobiotaDriven Activation of Intrahepatic B Cells Aggravates NASH Through Innate and Adaptive Signaling. Hepatology 2021;74:704-22.

8. Mauri C, Bosma A. Immune regulatory function of B cells. Annu Rev Immunol 2012;30:221-41.

9. Sutti S, Albano E. Adaptive immunity: an emerging player in the progression of NAFLD. Nat Rev Gastroenterol Hepatol 2020;17:81-92.

10. Miyake T, Abe M, Tokumoto $\mathrm{Y}$, et al. B cell-activating factor is associated with the histological severity of nonalcoholic fatty liver disease. Hepatol Int 2013;7:539-47.

11. Chen F, Esmaili S, Rogers GB, et al. Lean NAFLD: A Distinct Entity Shaped by Differential Metabolic Adaptation. Hepatology 2020;71:1213-27.

12. Barrow F, Khan S, Wang H, et al. The Emerging Role of B Cells in the Pathogenesis of NAFLD. Hepatology 2021;74:2277-86. 